\title{
Design Methodology for Quadcopter with Co-Axial Propulsion units to Achieve Thrust and Stability Demand During Flight
}

\author{
Vimalkumar. R \\ Dept. of Aerospace Engineering \\ SRM Institute of Science and Technology \\ Kattankulathur, Chengalpattu District, TamilNadu-603203, India.
}

\begin{abstract}
The usual Quadcopter has only four rotors and it achieves its Stability by nullifying the Rotational Moments produced by its corresponding rotors placed in the Frame.The addition of Co-Axial propulsion units, will leads to increase the Thrust, better Maneuverability and supports to achieve Stability in Emergency situations.A Quadcopter mounted CoAxial propulsion units will have less redundancy when compared to the Quadcopter having only four rotors, because the current requirement of 8 Brushless Direct Current Motor (BLDC) is comparatively larger than the current requirement of four motors so that the Flight time reduces for the Quadcopter having Co-Axial propulsion units.The usage of all Co-axial rotors produces lesser amount of Thrust as compared to the Thrust produced by only four rotors, because in CoAxial Propulsion units the lower propeller loses thrust working in prop wash of upper propeller but this offers ability to provide significantly higher thrust for the platform in smaller volume.During Emergency situations where the Failure of motor occurs, in this case the presence of Co-Axial propulsion units will help to produce corresponding Rotational moment that was produced by the motor which failed to work.In some case where all the four motors gets failed, the co-Axial propulsion units can be used as a Back-up and the mission can be completed.Though the Co-Axial propulsion units has some drawbacks, it can be used with additional battery as power source in the Quadcopter, not for continuous usage but can be used during demands.
\end{abstract}

Keywords-Quadcopter; Rotational Moment; Co-Axial Propulsion unit; Brushless Direct Current motor; propeller.

\section{INTRODUCTION}

The idea of coaxial rotors originates with Mikhail Lomonosov. He had developed a small helicopter model with coaxial rotors in July 1754 and demonstrated it to the Russian Academy of Sciences.In 1859, the British Patent Office awarded the first helicopter patent to Henry Bright for his coaxial design. From this point, coaxial helicopters developed into fully operational machines as we know them today.Two pioneering helicopters, the Corradino D'Ascanio-built "D'AT3" of 1930, and the generally more successful French mid-1930s Gyroplane Laboratoire, both used coaxial rotor systems for flight.

In the recent years the technological and mechanical areas in the field of Unmanned Aerial Vehicles were drastically developed.The Drone Technology is used in most of the civilian operations related to Agriculture, border Surveillance operations, inspection of critical areas, rescue operations and in fire control.The Propulsion systems of the
Drones is the most important component since it has higher power consumption. So that the propulsion systems must be efficient and appropriate to the mass of the Drone to have long Flight time.Additionally the propulsion units should produce higher Thrust so that the Thrust to weight ratio becomes significant to Lift the payload.

Propulsion units of the Drone are responsible for its survivability, endurance and other parameters crucial for its mission operation. Reliability is the main requirement of the Drone since the propulsion system failure inevitably leads to the Drone loss. The propulsion system should allow also a convenient drone climbing to high flight altitudes without power loss . A necessity to meet these requirements, as well as the special needs of specific applications, result in numerous technological and scientific challenges. This imposes a need for intensive research on UAV propulsion, which is constantly progressing and expanding.

\section{LITERATURE SURVEY}

B. Wang,Zhifei Hou,Wancen Wang.(2015) [1] Analyzed the hover duration performances of miniature VTOL(Vertical Takeoff and Landing) air vehicles depend on the performance of the propulsion system. Based on the mass models of battery, motor and ESC(Electronic Speed Controller), and the relationship between provided force and required power of propeller, the duration calculation model of vertical takeoff and landing air vehicles was established by using the battery constant-current discharge model. The influence of propulsion parameters and payload on vehicle duration is analyzed, which would be used for preliminary design of VTOL air vehicles and components selection of propulsion systems.

Wojciech ,Adam Bondyra, Stanisław Gardecki,Przemysław Gąsior.(2016) [2] proposed a contrast Experimental Analysis of different types of propulsion systems developed for multi rotor UAVs. One of the most interesting designs is the so called X8 quadcopter, which extends original quadrotor concept to 8 motors, arranged in 4 coaxial pairs.loss of efficiency due to coaxial propellers' configuration, because the lower propeller loses thrust working in prop wash of upper propeller. This paper presents the experimental verification of performance of such propulsion systems in practical terms of designing multi 
rotor platforms, compared to design with 8 isolated propulsion units. In addition, its advantages versus classic quadrotor concept is shown. The series of experiments with different motors and sizes of propellers were conducted to estimate efficiency of coaxial propulsion regarding useful thrust generated by each configuration.

Xunhua Dai,Quan Quan,Jinrui Ren,Kai-Yuan Cai.(2018) [3] proposed a practical method to help designers quickly select the optimal products of the propulsion system to maximize the multicopter efficiency under the desired flight condition. First, the modeling methods for the components of the propulsion system are studied respectively to describe the optimization problem mathematically. Secondly, methods are proposed to find optimal motor and propeller combination with the maximum thrust efficiency according to the given design requirements. Finally, factors that may affect the hovering time of multicopters are analyzed, and the optimal battery parameters are obtained for maximizing the multicopter endurance. Experiments and simulations are performed to demonstrate the effectiveness and practicability of the proposed method.

Wayne Ong,Spot Srigrarom,Henrik Hesse.(2019) [4] design methodology for multirotor Unmanned Aerial Vehicles (UAVs). To specifically address the design of vehicles with heavy lift capabilities, and have extended existing design methodologies to include coaxial rotor systems which have exhibited the best thrust-to-volume ratio for operation of UAVs in urban environments. Such coaxial systems, however, come with decreased aerodynamic efficiency and the design approach developed in this work can account for this. The proposed design methodology and included market studies have been demonstrated for the development of a multi-parcel delivery drone that can deliver up to four packages using a novel morphing concept. Flight test results in this paper serve to validate the predictions of thrust and battery life of the coaxial propulsion system suggesting errors in predicted flight time of less than 5 percent.

Marcin Biczyski,Rabia Sehab,James Whidborne,Patrick Chi Kwong Luk.(2020) [5] described the need for sizing of rotors for multirotor vehicle applications such as personal air transport, delivery, surveillance, and photography. A methodology for the propeller and motor selection is developed and augmented with flight time estimation capabilities. Being multirotor-specific it makes use of the platform's simplicity to rapidly provide a set of off-the-shelf components ready to be used in the vehicle. Use of operating points makes the comparison process fast, precise, and tailored to specific applications. The method is easily implemented in software to provide an automated tool. Furthermore, clearly defined input and output parameters make it also usable as a module in other multi criteria optimisation algorithms. The new methodology is validated through comparison with a consumer-grade drone and the calculated results are compliant with manufacturer's specification in terms of maximum hover time.

\section{CONSTRUCTION}

The prefix Quad-copter implies ("Quad" =four), is a drone configuration where there are four arms. The main frame is made of Carbon fiber due to its High Strength to Weight ratio, with each arm length of $230 \mathrm{~mm}$. At each free end of the arm, a pair of Co-Axial motors will be fixed and propeller will be mechanically coupled to the motor.For all eight motors the output side of an ESC will be connected and the input side of the ESC will be connected to the flight controller. The other input of the ESC will be connected to the power distribution board where the power supply is provided by the Li-Po battery. In a similar fashion all the other ESC's, motors and propellers are connected. A receiver will be connected to the Flight controller to receive signals from the transmitter. .The landing frame of height $140 \mathrm{~mm}$ is connected to the main frame such that there is enough space for payload attachment and the clearance for the propeller rotating down side.

\section{WORKING}

The signals will be transmitted from Transmitter and it will be received by the Receiver in the drone. From the receiver the signal goes to the Flight controller where the signal will be processed with accelerometer and gyroscope sensors. The processed signal will be sent to the ESC, which allows the specific amount to the motor based on the signal it receives. The propellers are mechanically coupled to the motors so that they rotate and produce thrust.The additional battery is mounted on the drone to provide power supply to the CoAxial Propulsion units.

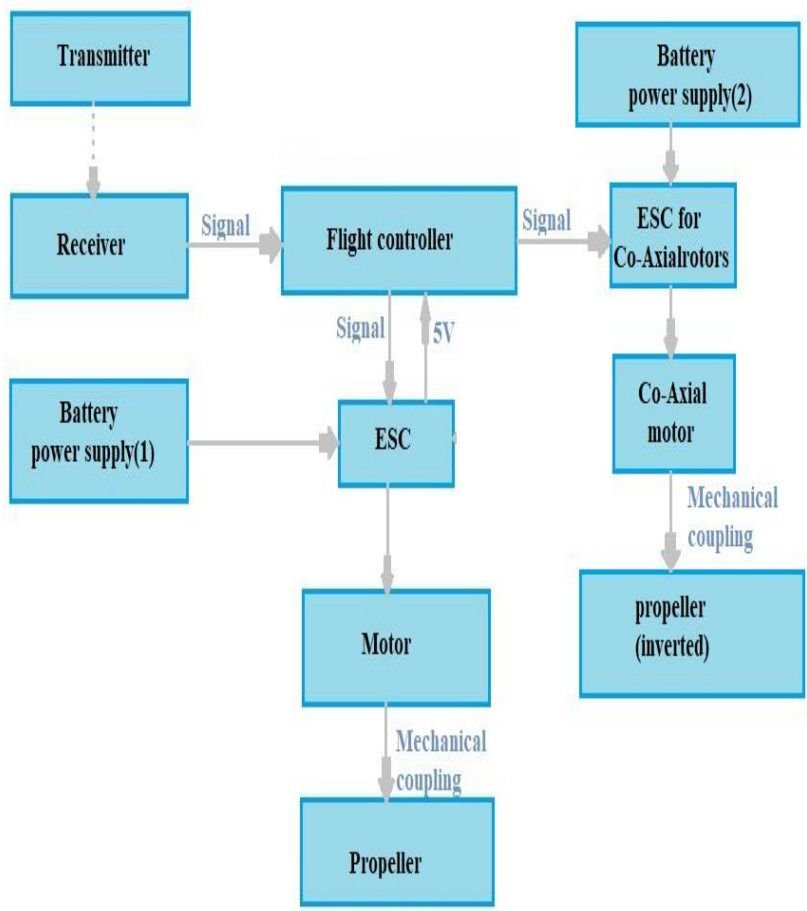

Figure 1 Block diagram of Working Process 


\section{COMPONENTS USED}

\section{A. MOTOR}

The Outer runner BLDC motors in which there are no brushes, they have a permanent magnet. Absence of brushes reduces the friction and enhances the work output of the motor.This BLDC motors can be controlled by varying the input current.This motor P2208 KV1750 produces 19,425 maximum Revolutions Per Minute(RPM) with 3s Li-Po battery and it produces a maximum thrust of 1457.83 grams with T5143s propeller.

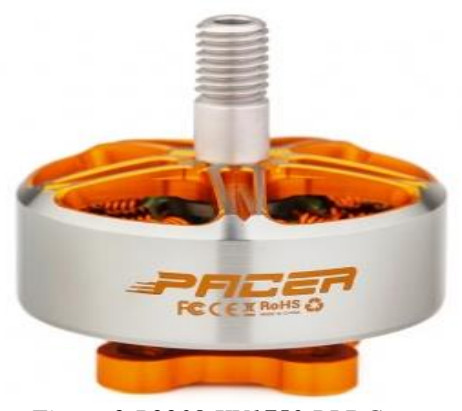

Figure 2 P2208 KV1750 BLDC motor

\section{B. PROPELLER}

The propeller has 3 blades with each blade of 5 inches length.It is made up of carbon fiber to have High strength to weight ratio and with features of Light weight, smoothness, High efficiency, Fast response and remarkable balance to the copter.

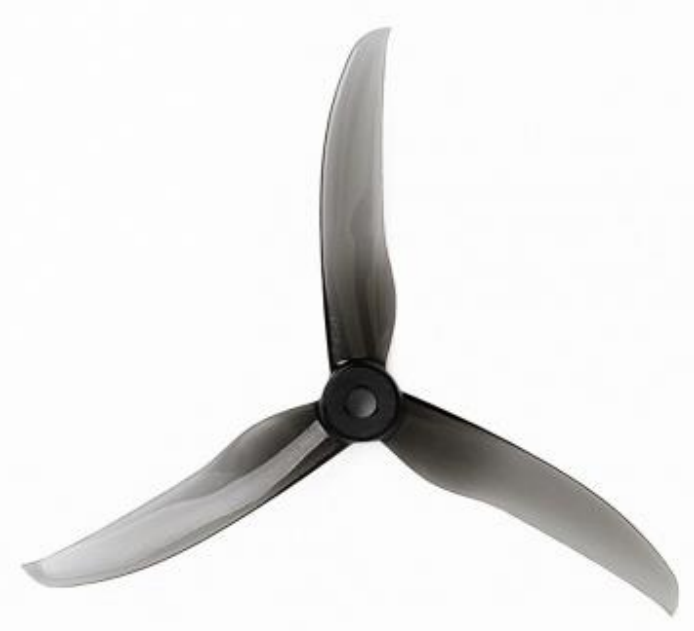

Figure 3 Propeller

\section{ESC}

It stands for Electronic Speed Controller and it is used to vary the RPM of the motor. 40A rated ESC is used asper the motor and battery specifications and this can handle upto $60 \mathrm{~A}$.

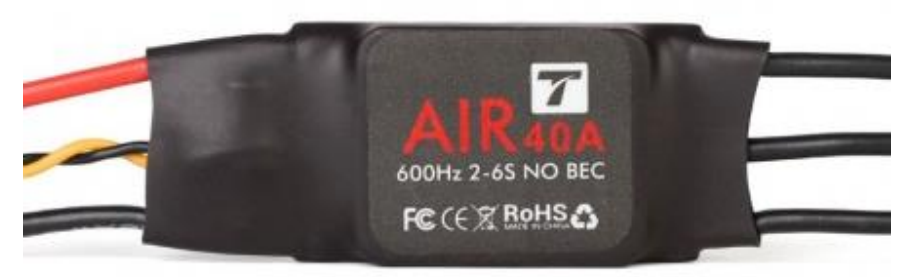

Figure 4 ESC

\section{BATTERY}

A 3 s Li-Po battery of $10000 \mathrm{mAh} 25 \mathrm{C}$ current capacity and $11.1 \mathrm{~V}$ is used to supply power to the motors at the topside of the Frame and another 3s Li-Po battery of $8000 \mathrm{mAh} 30 \mathrm{c}$ current capacity and $11.1 \mathrm{~V}$ is used to supply power to the motors at downside (co-Axial propulsion units). The current capacity of battery to supply power to the motors at topside is higher than the current capacity of the battery to power the motors at the down side, because the motors at downside will not be continuously operated and it will be operated in an Emergency situation, where the motors should respond quickly, so that the battery used has higher discharge rate of $30 \mathrm{c}$.

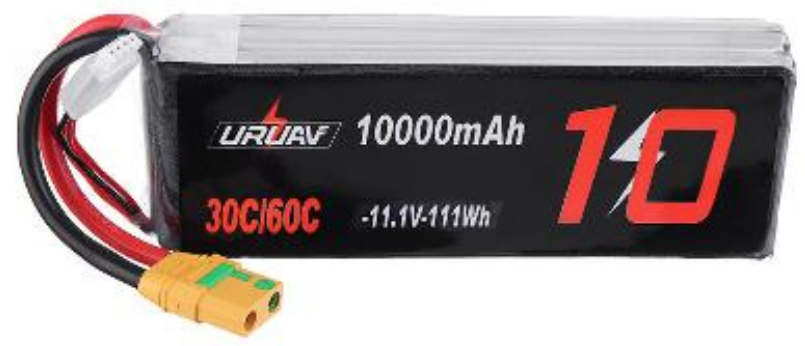

Figure 5 Battery to supply power for motors at the upper side

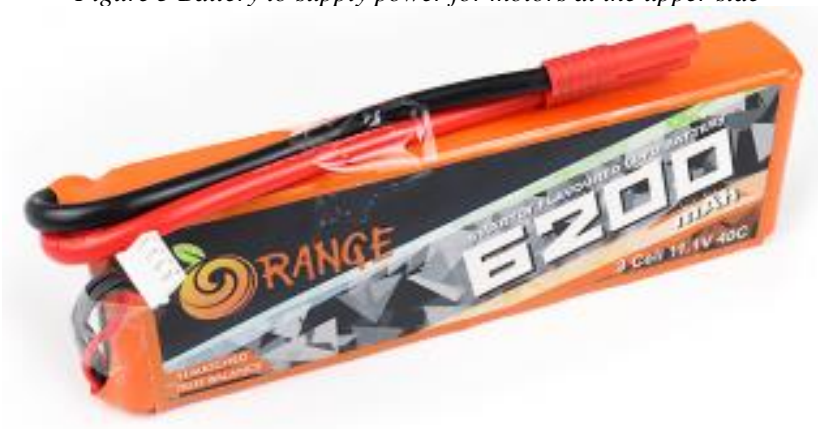

Figure 6 Battery to supply power for motors at the down side

\section{E. FLIGHT CONTROLLER AND RADIO CONTROLLERS}

Any Flight controller can be used but it has to be programmed in such a way that the usage of Co-Axial propulsion units are optional. The Transmitter used, should have additional options to engage and disengage the CoAxial propulsion units and the other options to operate a particular Co-Axial rotor in case of an Emergency situation where one of the rotors is not working. 
VI. WEIGHT BUILD-UP

\section{TABLE I COMPONENT WeIGHT DATA SHEET}

\begin{tabular}{|c|c|}
\hline PARTS & WEIGHT (grams) \\
\hline BLDC motor(8 No's) & 308.8 \\
\hline ESC (8 No's) & 208 \\
\hline Propeller(8 No's) & 30.4 \\
\hline Battery (for top side rotors) & 690 \\
\hline Battery (for down side rotors) & 615 \\
\hline $\begin{array}{l}\text { Receiver+Flight } \\
\text { controller+fastening components }\end{array}$ & 100 \\
\hline Frame & 300 \\
\hline TOTAL & 2251.14(approx) \\
\hline
\end{tabular}

The weight of the Flight controller, Receiver and other fastening components were taken into Approximation, since any kind of Flight controller and Receiver can be used in the Drone.The overall weight of the Drone is approximated as 2251.4 grams.

\section{G. THRUST CALCULATION}

Thrust to Weight ratio of the drone can be varied by engaging and disengaging the Co-Axial propulsion units. The additional rotors at the down side of the frame cannot be used continuously, because the lower propeller loses thrust working in prop wash of the upper propeller but this offers ability to provide significantly higher thrust for the platform in smaller volume.

Thrust to Weight ratio of the Drone when only the rotors at the top side is used,

Thrust produced with one motor and propeller combination $=1457.32$ grams

Total Thrust produced at $100 \% \mathrm{RPM}=1457.83 * 4$

$$
=5831.32 \mathrm{grams}
$$

Thrust to Weight Ratio $=$ Total Thrust produced $/$ Total weight of the drone

$$
\begin{aligned}
& =5831.22 / 2251.14 \\
& =2.50: 1
\end{aligned}
$$

Total Thrust produced at $100 \% \mathrm{RPM}(8$ motors $)=1457.83 * 8$

$=11662.64 \mathrm{grams}$

Thrust to Weight Ratio $=11662.64 / 2251.14$

$$
=5.18: 1
$$

The Thrust to Weight ratio can be increased about $107.2 \%$ but the additional Co-Axial propulsion units cannot be used continuously during Hovering, because the additional Thrust produced by them requires extra $17-29 \%$ power consumption to produce same amount of Thrust, as produced by the rotors at the top side.So this decreases the redundancy of the Drone. Instead of continuous usage of the Co-Axial rotors at the down side it can be used as a Back-up during an Emergency.

\section{H. BATTERY DRAIN TIME CALCULATION}

For a safer side, the battery drain time has to be calculated by considering the distance to be covered,Hovering Altitude and the time for the drone to return safely.

TABLE II CURRENT REQUIREMENT TABLE

\begin{tabular}{|c|c|}
\hline COMPONENTS & $\begin{array}{l}\text { CURRENT REQUIRED IN } \\
\text { (AMP) }\end{array}$ \\
\hline Motor(4 No's) & 124 \\
\hline ESC & 0.4 \\
\hline Receiver & 0.1 \\
\hline Flight controller & 0.1 \\
\hline TOTAL & $\mathbf{1 2 4 . 6}$ \\
\hline
\end{tabular}

A. BATTERY ENDURANCE FOR THE BATTERY THAT SUPPLY POWER TO THE MOTORS ON THE TOP SIDE,

Current output of the Battery $=10,000 \mathrm{mAh}$

Total current consumption of the components $=124.6 \mathrm{~A}$ (It includes current consumption of 4motors,4ESC,receiver and Flight controller)

Battery Endurance $=$ current output from battery/Total current consumption

$$
\begin{aligned}
& =10000 / 124.6 \mathrm{~A} \\
& =10 * 60 / 124.6 \mathrm{~A} \\
& =600 / 124.6 \mathrm{~A} \\
& =4.81 \text { minutes (at } 100 \% \text { Throttle) }
\end{aligned}
$$

\section{B. BATTERY ENDURANCE FOR THE BATTERY THAT SUPPLY} POWER TO THE MOTORS AT THE DOWN SIDE,

current output of the Battery $=6200 \mathrm{mAh}$

Total current consumption of the components $=124.4 \mathrm{~A}$ (It includes current consumption of 4motors, $4 \mathrm{ESC}$ )

Battery Endurance $=8000 / 124.4$

$$
\begin{aligned}
& =8.0 * 60 / 124.4 \\
& =480 / 124.4 \\
& =3.85 \text { minutes }(\text { at } 100 \% \text { Throttle) }
\end{aligned}
$$


Each motor at the down side requires about $17-29 \%$ more power to produce 5831.32 grams of Thrust.The Drone can be operated with only four rotors at it can have maximum Endurance as 4.81 minutes, after the battery drains the CoAxial propulsion units can be engaged and it can have maximum Endurance as 3.85 minutes.So the total Endurance of the Drone operated at this method can have Endurance as 8.66 minutes(at $100 \%$ RPM).

\section{STABILITY BACK-UP}

The presence of Co-Axial propulsion units helps with Stability Back-up. When there is a Failure of rotor during Flight, the quadcopter loses its Stability and then goes out of control.In this case where the Quadcopter mounted Coaxial propulsion units, the rotors can act as a Back-up to Produce the corresponding Rotational moment as produced by the rotor which was Failed.Hence the moments were restored and the Quadcopter will attain its Stability.

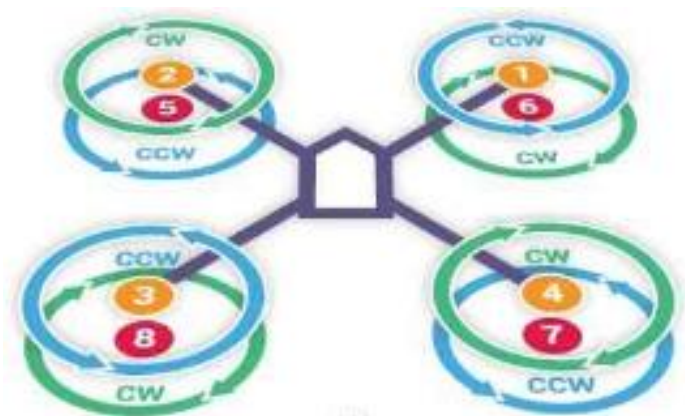

Figure 7 Quadcopter configuration labelled Rotors

The configuration of the Quadcopters with Co-Axial propulsion units appears as in Fig 7. ,if the rotor 2 labelled in the Fig 7. gets failed during Flight, then the corresponding clockwise moment will be produced by the rotor labelled 6 in the Fig 7 will start rotate and bring the Rotational moment of the Quadcopter to Equilibrium.

\section{STRUCTURAL DESCRIPTION}

The Main Frame of the Quadcopter is designed by determining the factors like Propeller dimensions, Clearance between two rotating propellers and the distance between Co-Axial propulsion units. Carbon Fiber can be used to Fabricate the Frame, because of its High strength to Weight Ratio.The Frame is designed in such a way that it can be assembled and disassembled easily. The CAD model of the Quadcopter shown in Fig8.is made using CATIA V5 software.

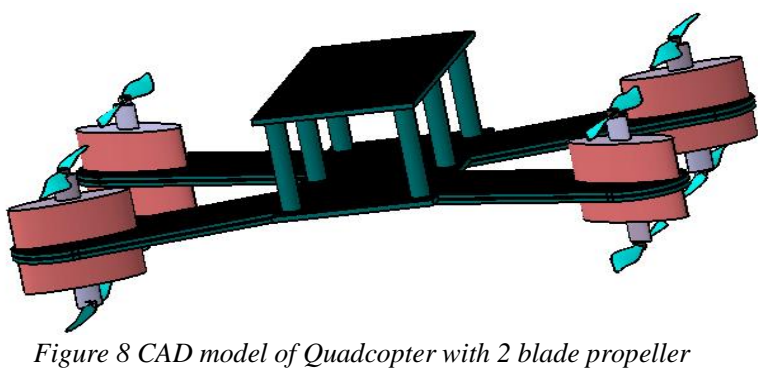

\section{CONCLUSION}

This paper describes the Advancement in the Quadcopter Configuration with Co-Axial propulsion units to produce additional thrust, provide Stability and better Maneuverability during Flight. This method of having additional rotors as a Back-up increases the Endurance when rotors are separately powered by two batteries and the Mission can be completed even with the Failure of some Rotors in the Quadcopter.

\section{REFERENCE}

[1] B. Wang,Zhifei Hou,Wancen Wang,"Performance analysis of propulsion system of miniature electric-powered vertical takeoff and landing air vehicles",Journal of National University of Defense Technology 37(3):84-90,June 2015

[2] Wojciech Giernacki,Adam Bondyra ,Stanisław Gardecki Przemysław Gąsior.'Performance of coaxial propulsion in design of multi-rotor UAVs",Advances in Intelligent Systems and Computing: Challenges in Automation, Robotics and Measurement Techniques,Edition: 440,Publisher: Springer International Publishing Switzerland,March 2016

[3] Xunhua Dai,Quan Quan,Jinrui Ren,Kai-Yuan Cai,"'An Analytical Design Optimization Method for Electric Propulsion Systems of Multicopter UAVs with Desired Hovering Endurance",IEEE/ASME Transactions on Mechatronics,Sep 2018

[4] Wayne Ong, Spot Srigrarom and Henrik Hesse,Design Methodology for Heavy-Lift Unmanned Aerial Vehicles with Coaxial Rotors",AIAA Scitech,Jan 2019

[5] Minwoo Kim,Byungwook Jang,Heejin Joo,’Conceptual multicopter sizing and performance analysis via component database",Ninth International Conference on Ubiquitous and Future Networks (ICUFN),July 2017

[6] Mantas Brazinskas,Stephen D Prior,James Scanlan,"An Empirical Study of Overlapping Rotor Interference for a Small Unmanned Aircraft Propulsion System",MDPI,Oct 2016

[7] Guang-Xun Du,Quan Quan,"Optimization of Multicopter Propulsion System Based on Degree of Controllability", journal of Aircraft 56(5):1-8,May 2018

[8] MoritzThiele,Martin Obster,Mirko Hornung."Aerodynamic Modeling of Coaxial Counter-Rotating UAV Propellers",8th Biennial Autonomous VTOL Technical Meeting,Feb 2019

[9] Wei-Liang Lyu,'Interactional Effect of PropulsivePropeller Location on Counter-RotatingCoaxial Main Rotor",JOURNAL OF AIRCRAFT,Vol. 55, No. 6, Dec 2018

[10] Yuening Jiang,"Aerodynamics Optimization of a Ducted Coaxial Rotor in Forward Flight Using Orthogonal Test Design"HindawiShock and VibrationVolume 2018, Article ID 2670439, May 2018

[11] Bart Theys,G. Dimitriadis,P. Hendrick,J. De Schutter,'Influence of propeller configuration on propulsion system efficiency of multirotor Unmanned Aerial Vehicles",2016 International Conference on Unmanned Aircraft Systems (ICUAS),June 2016 